\title{
Confirmatory factor analysis for Life Orientation Test-Revised among patients with esophageal cancer
}

\section{Authors}

Yangjun Liu, $\mathrm{MD}^{1}$; Erik Pettersson, $\mathrm{PhD}^{2}$; Asif Johar, $\mathrm{Msc}^{1}$; Pernilla Lagergren, $\mathrm{PhD}^{1,3}$

${ }^{1}$ Department of Molecular Medicine and Surgery, Karolinska Institutet, Karolinska University Hospital, Stockholm, Sweden

${ }^{2}$ Department of Medical Epidemiology and Biostatistics, Karolinska Institutet, Stockholm, Sweden

${ }^{3}$ Department of Surgery and Cancer, Imperial College London, London, UK

\section{Corresponding author}

Pernilla Lagergren, Department of Molecular Medicine and Surgery, Karolinska Institutet, Sweden (pernilla.lagergren@ki.se)

Note: This study has been published as a supplementary material of another study. Thus, if you want to cite the current study, please cite the published paper listed below:

Liu, Y., Pettersson, E., Schandl, A. et al. Psychological distress after esophageal cancer surgery and the predictive effect of dispositional optimism: a nationwide population-based longitudinal study. Support Care Cancer (2021). https://doi.org/10.1007/s00520-021-06517-x. 


\section{Supplementary Content 1}

\section{Confirmatory factor analysis for Life Orientation Test-Revised (LOT-R)}

\section{Introduction}

Dispositional optimism is commonly measured with the Life Orientation Test-Revised (LOTR), which consists of three positively worded items, three negatively worded items, and four filler items [1]. It is conceptualized as a continuous measure [2], and mostly treated as a bipolar unidimensional scale, with optimism and pessimism at the opposite end of a single spectrum [1,3]. However, some studies have also suggested that LOT-R is a bi-dimensional scale, with two partially independent factors, namely, optimism and pessimism [4-7]. Nevertheless, some researchers have argued that the two-factor structure might be attributed to a methodological artefact [8-10], and proposed a revised unidimensional structure, where one general factor represents dispositional optimism, on which all items load, and one method factor includes the positively (or negatively) worded items [8-11].

Given the lack of consensus about the dimensionality of the LOT-R, and that no study to date has assessed the psychometric property of LOT-R among patients with esophageal cancer, we conducted confirmatory factor analysis (CFA) for LOT-R based on our Swedish nationwide population-based data.

\section{Methods}

\section{Participants}

Data for the present analysis is from a prospective, ongoing Swedish-nationwide cohort study entitled Oesophageal Surgery on Cancer patients - Adaptation and Recovery (OSCAR). Detailed description of the OSCAR study can be found elsewhere [12]. In total, 197 patients 
who underwent esophageal cancer surgery between January 1, 2013 and December 31, 2016 and answered the LOT-R were included in the analysis.

\section{Swedish version of LOT-R}

The Swedish version of LOT-R scale has been validated, and consists of six items (the four filler items were removed) [13]. Patients were asked to report their agreement with each 5point Likert item, ranging from 0 ("strongly disagree") to 4 ("strongly agree") [1].

\section{Statistical Analysis}

We conducted descriptive analysis and examined the distribution of response to each item. Additionally, we calculated the correlation between all items. CFA with robust maximum likelihood estimation (MLR) was used to assess the dimensionality of LOT-R. We evaluated the model fit of four potential models reported in previous studies (sFigure 1). Model 1 was a congeneric one-factor model positing one dispositional optimism factor without any correlated uniqueness. Model 2 was an oblique two-factor model presuming optimism and pessimism are two correlated factors. Model 3 assumed one general factor (dispositional optimism) plus a method factor associated with positively worded item, while model 4 contained one general factor (dispositional optimism) plus a method factor associated with reversed negatively worded items. Model fit was assessed by $\chi 2$ test, root mean square error of approximation (RMSEA), standardized root mean square residual (SRMR), comparative fit index (CFI), and Akaike's information criterion (AIC). RMSEA and SRMR are absolute indices, with RMSEA value $<0.05$ and SRMR value $<0.08$ indicating good overall model fit [14]. CFI compares the target model with the baseline model, and value $>0.95$ represents good model fit [14]. AIC evaluates both goodness of fit and parsimony, and the model with lower AIC value is preferred [15]. Nested models were compared by the Satorra-Bentler scaling correction likelihood ratio $(\Delta \chi 2)$ test [16]. 
In addition, we estimated internal reliability, which is the variance in the observed measure that can be attributed to a corresponding and unobserved latent construct. We adopted McDonald's omega $(\omega)$ as the indicator, because it is based on the actual measurement model and allows items to load freely on factors [17]. Omega total $\left(\omega_{t}\right)$ is the variance ratio explained by both the general factor and the method factor, whereas omega hierarchical $(\omega \mathrm{h})$ is the variance ratio explained only by the general factor [17]. We calculated $\omega_{\mathrm{t}}$ for all models and $\omega_{\mathrm{h}}$ for the models with method factor. We used bootstrap to compute the $95 \%$ confidence intervals (CIs) for $\omega$. All analyses were performed using Mplus version 8.2 (Muthén \& Muthén, Los Angeles, USA).

\section{Results}

\section{Patients' response to each item}

Descriptive statistics of patients' responses to each item are displayed in sTable 1. Among the included 197 patients, 13 (7\%) patients responded to part of the items, and most missing (8 patients, 4\%) occurred in the first negatively worded item (item N2, "if something can go wrong for me, it will”). Furthermore, compared with other items, response to item N2 had unusually large variation. sFigure 2 displays the distribution of patients' response to each item. All responses were skewed to one direction (more positive or less negative) except for item N2, which was bimodal. Moreover, item N2 had positive correlation with both positively and negatively worded items (sTable 2).

\section{Model fit and omega value for potential models of LOT-R with six items}

The fit indices for the assessed four potential models are presented in sTable 3 . The model 4 assuming one general factor (dispositional optimism) plus a method factor associated with reversed negatively worded items had the lowest AIC, and fit the data best, with Yuan-Bentler $\chi^{2}(6)=8.158(\mathrm{p}=0.227), \mathrm{CFI}=0.983, \mathrm{RMSEA}=0.043$ and $\mathrm{SRMR}=0.039$. These results 
indicated that LOT-R was unidimensional, but with an artificial method factor associated with reversed negatively worded items. However, the loading of item N2 in this model was negative even though the score has already been reversed to account for the negative wording (sTable 4). In addition, McDonald's omega values for the four models were not satisfying (range: $\omega_{\mathrm{t}} 0.494$ to 0.578 , $\omega_{\mathrm{h}}: 0.285$ to 0.492 ). In model 4 , which showed the best fit, the ratio of total variance explained by general factor (dispositional optimism) was 0.285 , with $95 \%$ bootstrapped CI from 0.108 to 0.427 (sTable 3).

\section{Removal of the first negatively worded item (item N2)}

The above results showed that patients' response to the first negatively worded item (item N2) in LOT-R was bimodal, and it had equivocal correlation with both positively worded items and negatively worded items. Moreover, its loading was negative in the best-fit model even though it has already been reversed to account for the negative wording. Because it is the first negatively worded item in the LOT-R scale, patients might misunderstand the wording and scoring method, which could cause the bimodal distribution of the response and ensuing large variation and negative loading. In addition, patients filled in the LOT-R during an on-site interview, and they could check with the research nurse if they found any item unclear. However, only few patients expressed confusion about the LOT-R and they did not express specific confusion about the first negatively worded item. Furthermore, this item had the largest percentage of missingness, and patient representatives in our research partnership group reported that the hypothetical wording ("If something can go wrong for me, it will") reminded cancer recurrence to some extent. Thus, there might be another possibility that patients were confused on this item but they were reluctant to check with the research nurse due to accompanying uncomfortable feeling or social desirability, or patients answered this item not based on their generalized expectations for future outcomes but specific worries about cancer recurrence or death. In order to remove the noise caused by this item, we 
excluded it and reassessed the model fit of four similar models based on the remaining five items (model diagrams were presented in sFigure 3).

\section{Model fit and omega value for potential models of LOT-R with five items}

The model fit for the four rebuilt models are displayed in sTable 5. The model 3 with one general factor (dispositional optimism) plus a method factor associated with positively worded items showed poor RMSEA (0.111) and the null hypothesis that the model fit the data was rejected (Yuan-Bentler $\chi 2(2)=6.858, p=0.032$ ), even though this model had good CFI (0.953) and SRMR (0.018) values, and relatively low AIC (2735.815). The other two models, oblique two-factor model (model 2) and one general factor (dispositional optimism) model with correlated errors between reversed negatively worded items (model 4) showed the same best model fit (Yuan-Bentler $\chi^{2}(4)=5.854, p=0.210 ; \mathrm{AIC}=2734.293 ; \mathrm{CFI}=0.982$; RMSEA $=0.049 ;$ SRMR $=0.035)$. After removing the first negatively worded item, McDonald's omega values for all reassessed models were increased (range: $\omega_{\mathrm{t}} 0.568-0.724$, Wh: $0.486-0.496)$.

Because the one-dimensional bi-polar model has a stronger theoretical base [2], we chose the one general factor (dispositional optimism) model with correlated errors between reversed negatively worded items (model 4) in the present study, and the total variance explained by the general factor (dispositional optimism) was 0.486 ( $95 \%$ bootstrapped CI: 0.306 to 0.616 ). 


\section{References}

1. Scheier MF, Carver CS, Bridges MW (1994) Distinguishing optimism from neuroticism (and trait anxiety, self-mastery, and self-esteem): a reevaluation of the Life Orientation Test. J Pers Soc Psychol 67 (6):1063-1078. doi:10.1037//0022-3514.67.6.1063

2. Carver CS, Scheier MF, Segerstrom SC (2010) Optimism. Clinical psychology review 30 (7):879-889. doi:10.1016/j.cpr.2010.01.006

3. Mehrabian A, Ljunggren E (1997) Dimensionality and content of optimism-pessimism analyzed in terms of the pad temperament model. Personality and Individual Differences 23 (5):729-737. doi:10.1016/s0191-8869(97)00119-0

4. Herzberg PY, Glaesmer H, Hoyer J (2006) Separating optimism and pessimism: a robust psychometric analysis of the revised Life Orientation Test (LOT-R). Psychol Assess 18 (4):433-438. doi:10.1037/1040-3590.18.4.433

5. Glaesmer H, Rief W, Martin A, Mewes R, Brahler E, Zenger M, Hinz A (2012) Psychometric properties and population-based norms of the Life Orientation Test Revised (LOT-R). British journal of health psychology 17 (2):432-445. doi:10.1111/j.20448287.2011.02046.x

6. Zenger M, Finck C, Zanon C, Jimenez W, Singer S, Hinz A (2013) Evaluation of the Latin American version of the Life Orientation Test-Revised. Int J Clin Hlth Psyc 13 (3):243-252. doi:10.1016/s1697-2600(13)70029-2

7. Creed PA, Patton W, Bartrum D (2002) Multidimensional Properties of the Lot-R: Effects of Optimism and Pessimism on Career and Well-Being Related Variables in Adolescents. Journal of Career Assessment 10 (1):42-61. doi:10.1177/1069072702010001003 8. Rauch WA, Schweizer K, Moosbrugger H (2007) Method effects due to social desirability as a parsimonious explanation of the deviation from unidimensionality in LOT-R scores. Personality and Individual Differences 42 (8):1597-1607. doi:10.1016/j.paid.2006.10.035 
9. Vautier S, Raufaste E, Cariou M (2003) Dimensionality of the Revised Life Orientation Test and the status of filler items. International Journal of Psychology 38 (6):390-400. doi:10.1080/00207590344000222

10. Vecchione M, Alessandri G, Caprara GV, Tisak J (2014) Are Method Effects Permanent or Ephemeral in Nature? The Case of the Revised Life Orientation Test. Structural Equation Modeling: A Multidisciplinary Journal 21 (1):117-130. doi:10.1080/10705511.2014.859511 11. Pan TM, Mills SD, Fox RS, Baik SH, Harry KM, Roesch SC, Sadler GR, Malcarne VL (2017) The Psychometric Properties of English and Spanish Versions of the Life Orientation Test-Revised in Hispanic Americans. J Psychopathol Behav Assess 39 (4):657-668. doi:10.1007/s10862-017-9606-x

12. Schandl A, Johar A, Anandavadivelan P, Vikstrom K, Malberg K, Lagergren P (2020) Patient-reported outcomes 1 year after oesophageal cancer surgery. Acta Oncol 59 (6):613619. doi:10.1080/0284186X.2020.1741677

13. Muhonen T, Torkelson EVA (2005) Kortversioner av frågeformulär inom arbets- och hälsopsykologi—om att mäta coping och optimism. Nordisk Psykologi 57 (3):288-297. doi:10.1080/00291463.2005.10637375

14. Hu Lt, Bentler PM (1999) Cutoff criteria for fit indexes in covariance structure analysis: Conventional criteria versus new alternatives. Structural Equation Modeling: A Multidisciplinary Journal 6 (1):1-55. doi:10.1080/10705519909540118 15. Vandekerckhove J, Matzke D, Wagenmakers E-J (2015) Model comparison and the principle of parsimony. In: The Oxford handbook of computational and mathematical psychology. Oxford library of psychology. Oxford University Press, New York, NY, US, pp $300-319$

16. Satorra A, Bentler PM (2010) Ensuring Positiveness of the Scaled Difference Chi-square Test Statistic. Psychometrika 75 (2):243-248. doi:10.1007/s11336-009-9135-y 
17. Revelle W, Condon DM (2019) Reliability from alpha to omega: A tutorial. Psychol Assess 31 (12):1395-1411. doi:10.1037/pas0000754 
sTable 1. Descriptive statistics for the six items of Life Orientation Test-Revised answered by 197 patients with esophagectomy for cancer

\begin{tabular}{cccccc}
\hline Item & Sample size & Mean & Variance & Skewness & Kurtosis \\
\hline P1 & 191 & 3.052 & 0.992 & -0.836 & 0.149 \\
P3 & 197 & 3.269 & 0.846 & -1.143 & 0.715 \\
P6 & 192 & 3.115 & 1.174 & -1.358 & 1.268 \\
N2 & 189 & 2.053 & 2.241 & -0.128 & -1.455 \\
N4 & 193 & 1.358 & 1.307 & 0.539 & -0.557 \\
N5 & 194 & 1.113 & 1.265 & 0.819 & -0.198 \\
\hline
\end{tabular}

Note. P1, P2, and P3 represent positively worded items 1, 3, and 6, respectively. N2, N4 and N5 represent negatively worded items 2,4 and 5, respectively. 
sTable 2. Item intercorrelations in Life Orientation Test-Revised

\begin{tabular}{ccccccc}
\hline & P1 & P3 & P6 & N2 & N4 & N5 \\
\hline P1 & 1.000 & & & & & \\
P3 & 0.400 & 1.000 & & & & \\
P6 & 0.300 & 0.303 & 1.000 & & & \\
N2 & $\mathbf{0 . 2 0 9}$ & $\mathbf{0 . 1 0 5}$ & $\mathbf{0 . 0 1 4}$ & $\mathbf{1 . 0 0 0}$ & & \\
N4 & -0.089 & -0.074 & -0.218 & $\mathbf{0 . 2 3 2}$ & 1.000 & \\
N5 & -0.147 & -0.207 & -0.223 & $\mathbf{0 . 2 3 4}$ & 0.548 & 1.000 \\
\hline
\end{tabular}

Note. P1, P2, and P3 represent positively worded items 1, 3, and 6, respectively. N2, N4 and N5 represent negatively worded items 2,4 and 5 , respectively. 
sTable 3. Fit statistics and McDonald's omega for the assessed four potential structures of Life Orientation Test-Revised (six items)

\begin{tabular}{|c|c|c|c|c|c|c|c|c|c|}
\hline $\begin{array}{l}\text { Model } \\
\text { (6 items) }\end{array}$ & $\begin{array}{c}\mathrm{Y}-\mathrm{B} \chi^{2} \\
(d f)\end{array}$ & $p$ & AIC & CFI & RMSEA & SRMR & $\Delta \chi 2_{\text {s-в }}(d f)$ & $p$ & $\begin{array}{l}\text { McDonald's omega } \\
\text { ( } 95 \% \text { bootstrapped CI) }\end{array}$ \\
\hline
\end{tabular}

1. Congeneric one-factor model

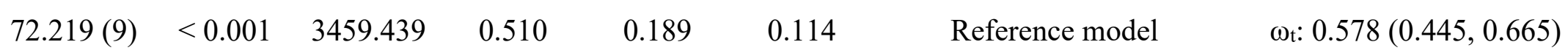

2. Oblique two-factor model

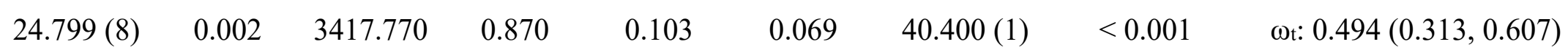

3. One general factor (dispositional optimism) plus a method factor associated with positively worded items

$$
\begin{array}{rllllllll}
24.929(6) & <0.001 & 3418.561 & 0.853 & 0.127 & 0.063 & 38.447(3) & <0.001 & \omega_{h}: 0.492(0.378,0.583)
\end{array}
$$

$\omega_{\mathrm{t}}: 0.664(0.570,0.720)$

4. One general factor (dispositional optimism) plus a method factor associated with reversed negatively worded items

$$
\begin{array}{lllllllll}
8.158(6) & 0.227 & 3408.122 & 0.983 & 0.043 & 0.039 & 85.001(3) & <0.001 & \omega_{\mathrm{t}:} 0.640(0.532,0.712) \\
\omega_{h}: 0.285(0.108,0.427)
\end{array}
$$

Note. Y-B: Yuan-Bentler; df: degrees of freedom; p: p value; AIC: Akaike information criterion; CFI: comparative fit index; RMSEA: root mean square error of approximation; SRMR: standardized root mean square residual; CI: confidence interval; $\omega_{\mathrm{t}}$ : McDonald's omega total; $\omega_{\mathrm{h}}$ : McDonald's omega hierarchical. 
sTable 4. Loadings of the six items of Life Orientation Test-Revised in the model assuming one general factor (dispositional optimism) plus a method factor associated with reversed negatively worded items

\begin{tabular}{lll}
\hline Item & Unstandardized factor loading $\quad$ Standardized factor loading \\
\hline
\end{tabular}

\section{General factor (dispositional optimism)}

P1: In uncertain times, I usually expect the best.

$$
1.000 \quad 0.644
$$

P3: I am always optimistic about my future.

P6: Overall, I expect more good things to happen to me than bad.

$$
0.814
$$

re_N2: If something can go wrong for me, it will.

$$
-\mathbf{0 . 5 0 3}
$$

re_N4: I hardly ever expect things to go my way.

$$
0.337
$$

re_N5: I rarely count on good things happening to me.

$$
0.538
$$

\section{Method factor}

re_N2: If something can go wrong for me, it will.

$$
1.000
$$

re_N4: I hardly ever expect things to go my way.

$$
1.250
$$

re_N5: I rarely count on good things happening to me.

$$
1.352
$$

0.828

Note. P1, P2, and P3 represent positively worded items 1, 3, and 6, respectively. N2, N4 and N5 represent negatively worded items 2, 4 and 5, respectively. re_N2, re_N4 and re_N5 represent reversed negatively worded items 2,4 and 5, respectively. 
sTable 5. Fit statistics and McDonald's omega for the reassessed four potential structures of Life Orientation Test-Revised (five items)

\begin{tabular}{|c|c|c|c|c|c|c|c|c|c|}
\hline $\begin{array}{l}\text { Model } \\
\text { (5 items) }\end{array}$ & $\begin{array}{c}\mathrm{Y}-\mathrm{B} \chi^{2} \\
(d f)\end{array}$ & $p$ & AIC & CFI & RMSEA & SRMR & $\Delta \chi 2_{\text {s-в }}(d f)$ & $p$ & $\begin{array}{l}\text { McDonald's omega } \\
\text { ( } 95 \% \text { bootstrapped CI) }\end{array}$ \\
\hline
\end{tabular}

1. Congeneric one-factor model

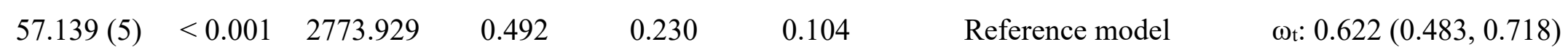

2. Oblique two-factor model

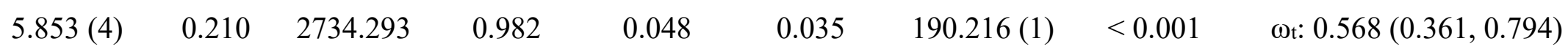

3. One general factor (dispositional optimism) plus a method factor associated with positively worded items

$$
\begin{array}{lllllllll}
6.858(2) & 0.032 & 2735.815 & 0.953 & 0.111 & 0.018 & 41.450(3) & <0.001 & \omega_{h}: 0.496(0.349,0.593)
\end{array}
$$

$\omega_{\mathrm{t}}: 0.724(0.568,0.780)$

4. One general factor (dispositional optimism) model with correlated errors between reversed negatively worded items

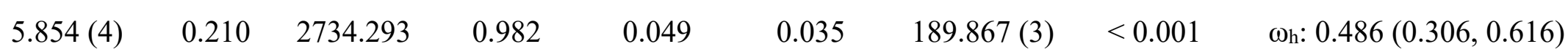

Note. Y-B: Yuan-Bentler; df: degrees of freedom; p: p value; AIC: Akaike information criterion; CFI: comparative fit index; RMSEA: root mean square error of approximation; SRMR: standardized root mean square residual; CI: confidence interval; $\omega_{\mathrm{t}}$ : McDonald's omega total; $\omega_{\mathrm{h}}$ : McDonald's omega hierarchical. 


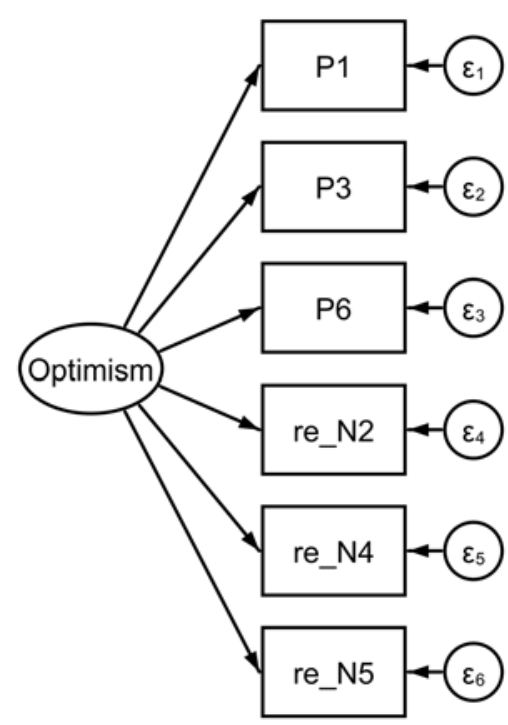

Model 1. Congeneric one-factor model

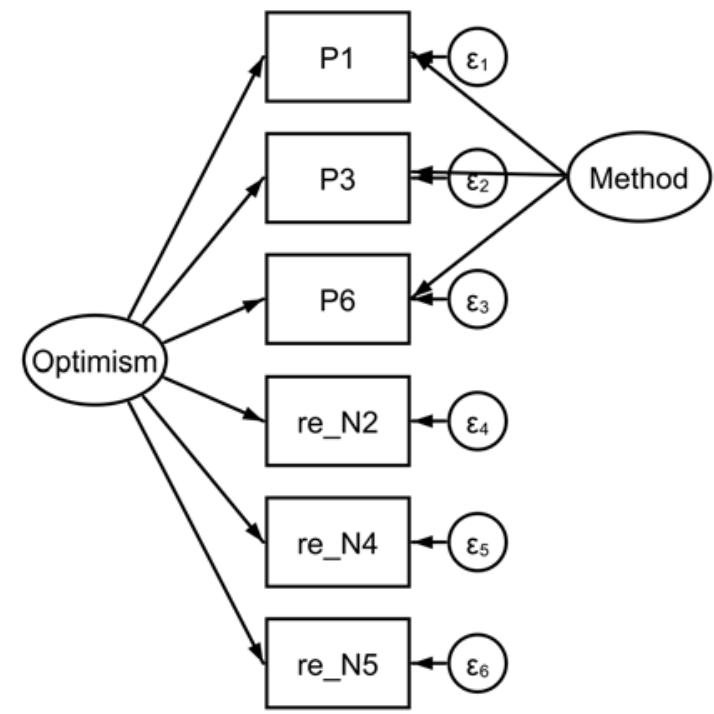

Model 3. One general factor (dispositional optimism) plus a method factor associated with positively worded items

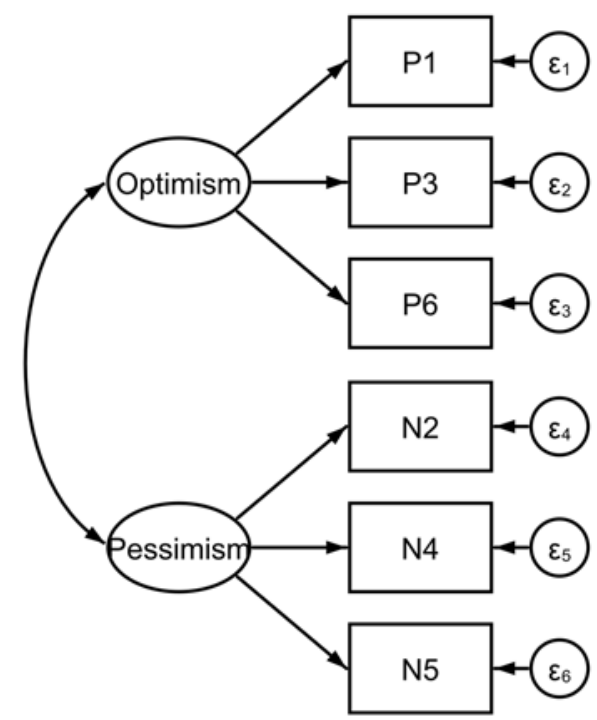

Model 2. Oblique two-factor model

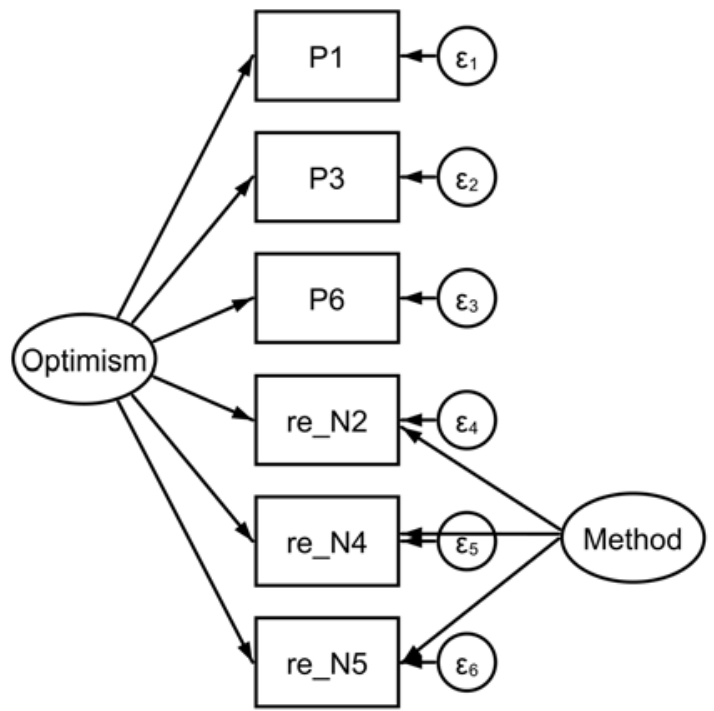

Model 4. One general factor (dispositional optimism) plus a method factor associated with reversed negatively worded items

sFigure 1. Assessed models for Life Orientation Test-Revised (six items)

Note. P1, P2, and P3 represent positively worded items 1, 3, and 6, respectively. N2, N4 and N5 represent negatively worded items 2, 4 and 5, respectively. re_N2, re_N4 and re_N5 represent reversed negatively worded items 2, 4 and 5, respectively. 


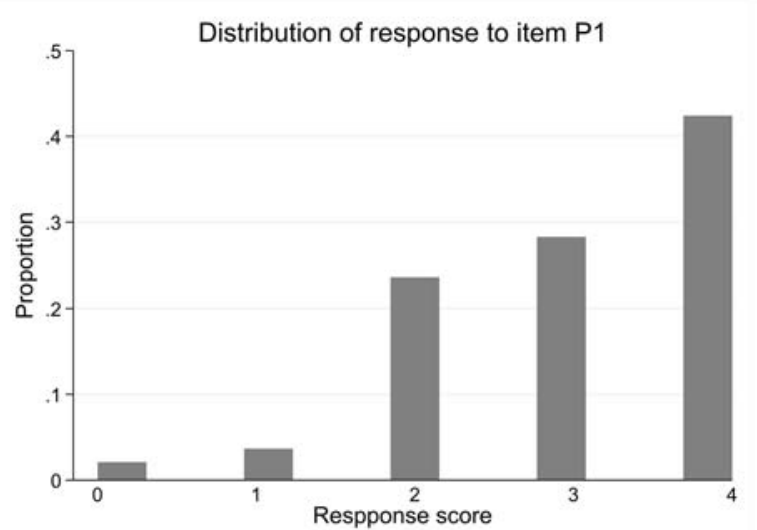

Respponse score

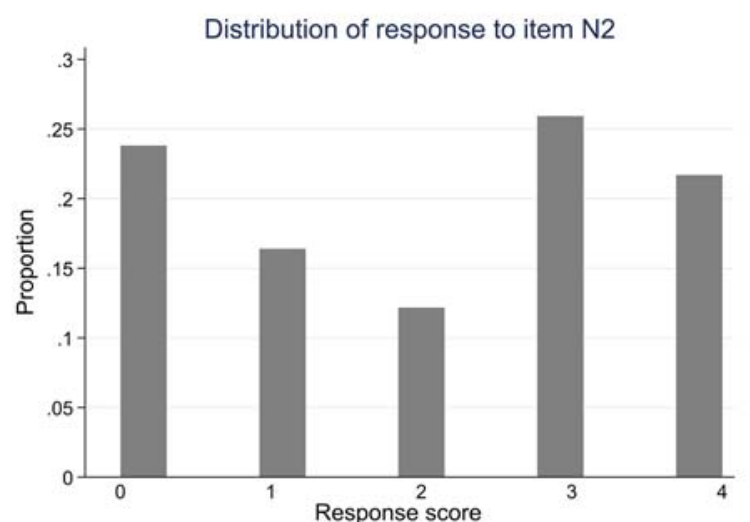

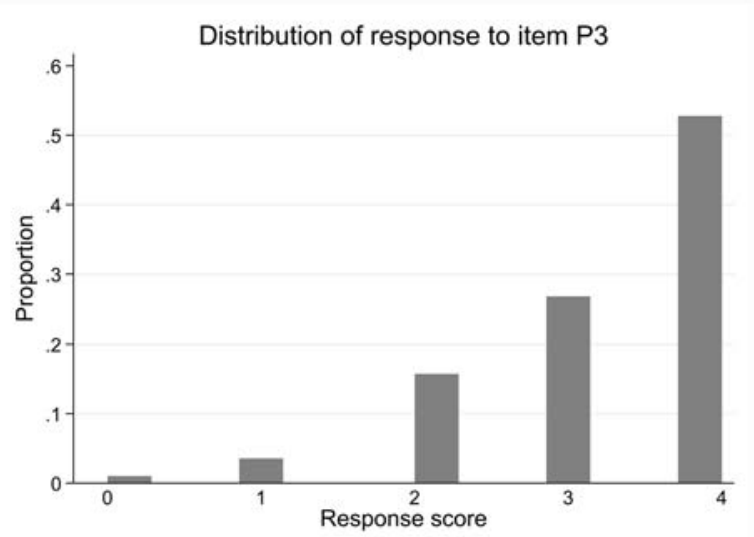

Distribution of reponse to item N4

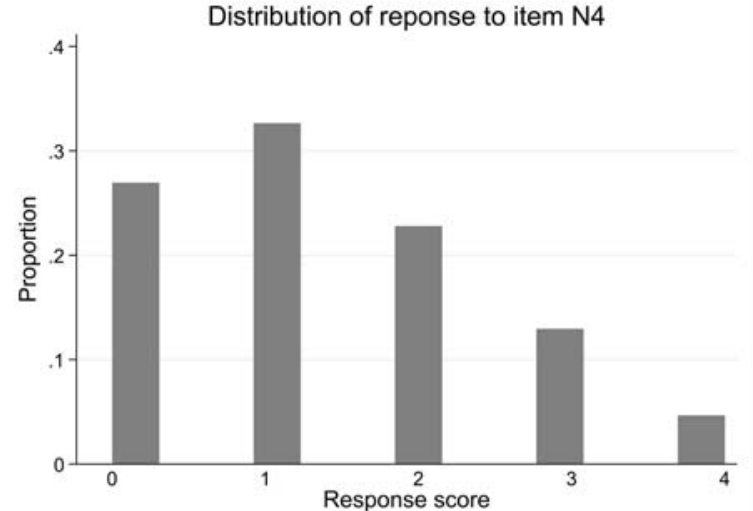

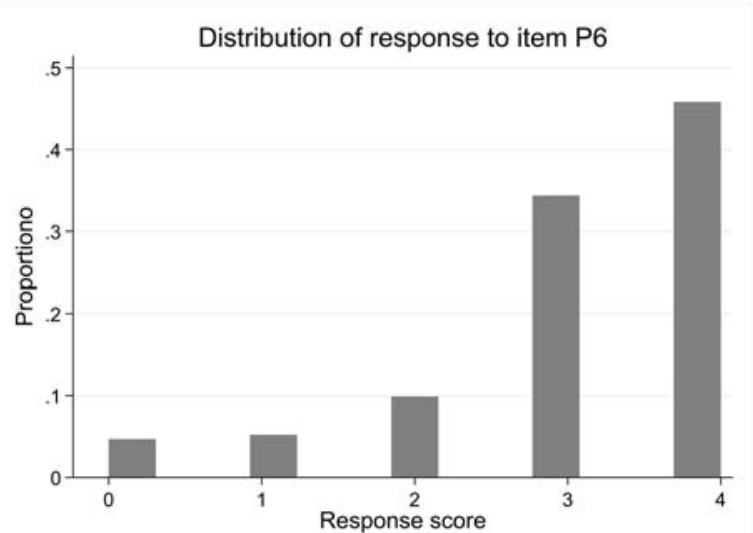

Distribution of response to item N5

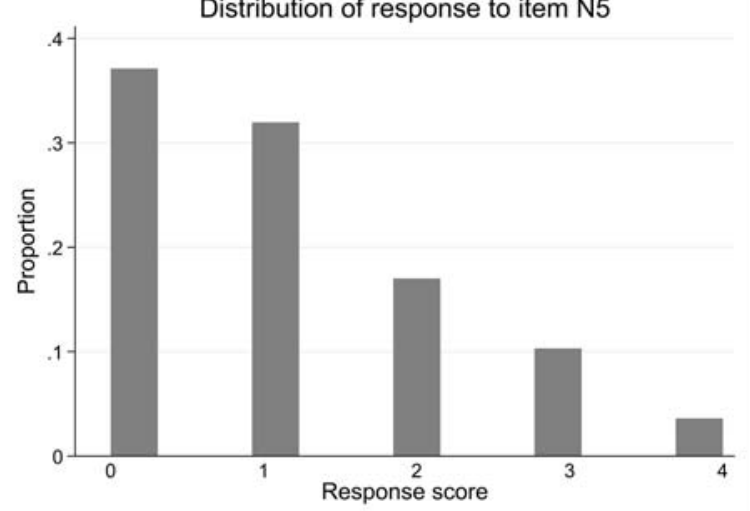

sFigure 2. Distribution of response to each item in Life Orientation Test-Revised

Note. P1, P2, and P3 represent positively worded items 1, 3, and 6, respectively. N2, N4 and N5 represent negatively worded items 2, 4 and 5, respectively. Score 0: "I disagree a lot"; score 1: "I disagree a little"” score 2: "I neither agree nor disagree"; score 3: "I agree a little"; score 4: "I agree a lot". 


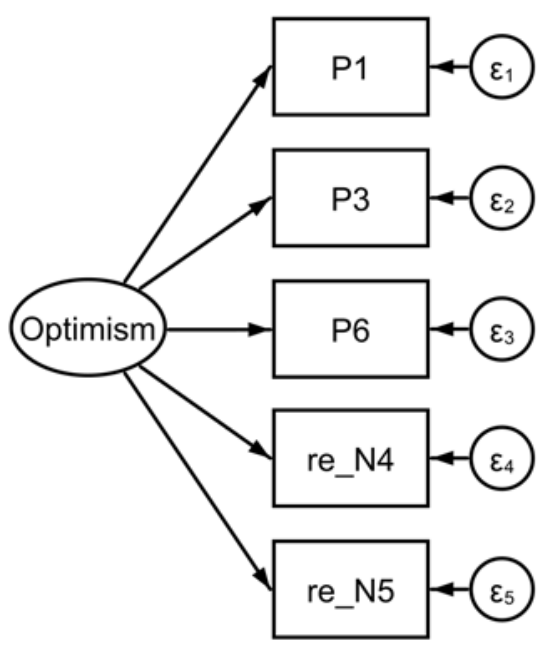

Model 1. Congeneric one-factor model

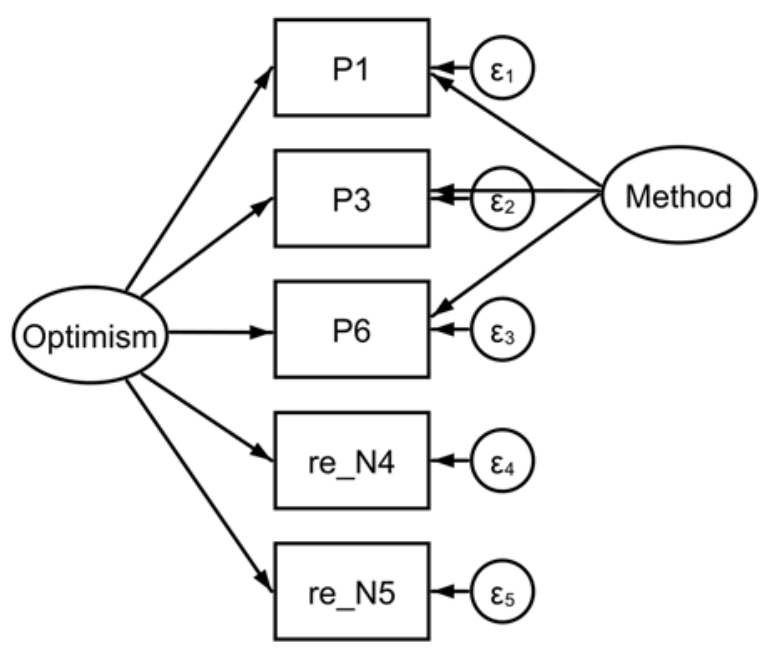

Model 3. One general factor (dispositional optimism) plus a method factor associated with positively worded items

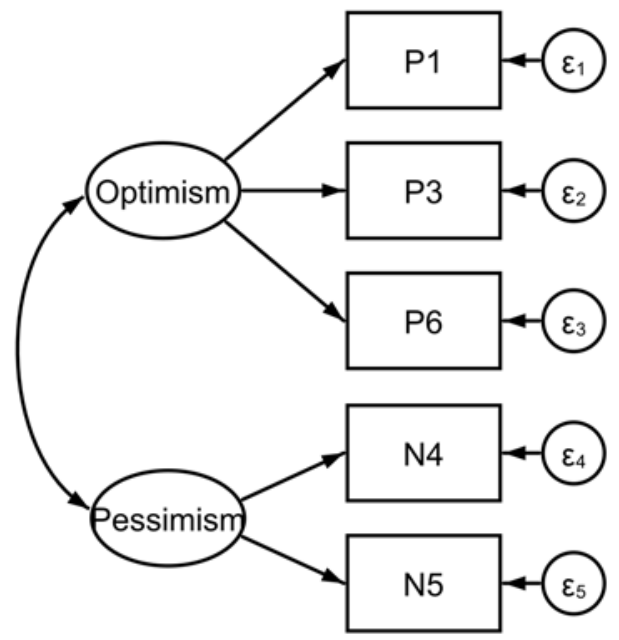

Model 2. Oblique two-factor model

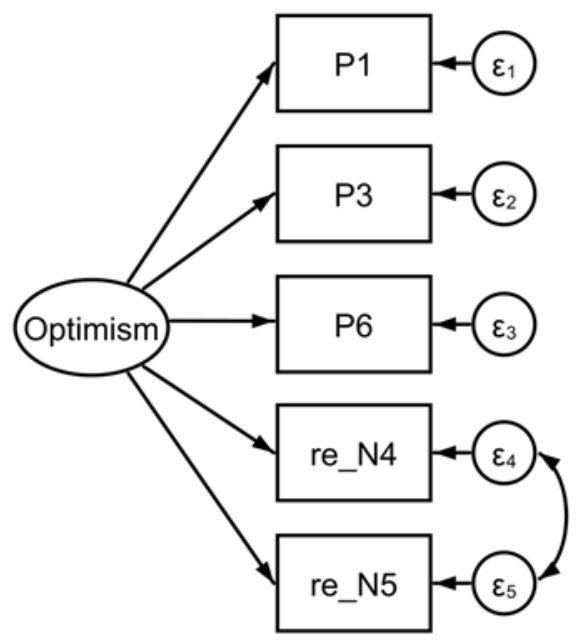

Model 4. One general factor (dispositional optimism) model with correlated errors between reversed negatively worded items

sFigure 3. Reassessed models for Life Orientation Test-Revised (five items).

Note. P1, P2, and P3 represent positively worded items 1, 3, and 6, respectively. N2, N4 and N5 represent negatively worded items 2, 4 and 5, respectively. re_N2, re_N4 and re_N5 represent reversed negatively worded items 2, 4 and 5, respectively. 